\title{
Preserving and Delivery Systems of Bioactives and Functional Compounds of Chia Seed (Salvia hispanica L.) ${ }^{\dagger}$
}

\author{
Luciana M. Julio $^{1}$, Claudia N. Copado ${ }^{1}$, Vanesa Y. Ixtaina ${ }^{1,2}$ and Mabel C. Tomás ${ }^{1, *(D)}$ \\ 1 Centro de Investigación y Desarrollo en Criotecnología de Alimentos (CIDCA), CCT-CONICET, CICPBA, \\ Universidad Nacional de La Plata (UNLP), 47 y 116, La Plata 1900, Argentina; \\ luci_julio86@hotmail.com (L.M.J.); claudiacopado16@gmail.com (C.N.C.); vanesaix@hotmail.com (V.Y.I.) \\ 2 Facultad de Ciencias Agrarias y Forestales (FCAyF, UNLP), 60 y 119, La Plata 1900, Argentina \\ * Correspondence: mabtom@hotmail.com or mtomas@quimica.unlp.edu.ar \\ + Presented at the III Conference la ValSe-Food and VI Symposium Chia-Link Network, Online, \\ 15-17 November 2021.
}

Citation: Julio, L.M.; Copado, C.N.; Ixtaina, V.Y.; Tomás, M.C. Preserving and Delivery Systems of Bioactives and Functional Compounds of Chia Seed (Salvia hispanica L.). Biol. Life Sci. Forum 2021, 8, 11. https://doi.org/ $10.3390 /$ blsf2021008011

Academic Editors: Loreto Muñoz and Claudia M. Haros

Published: 25 January 2022

Publisher's Note: MDPI stays neutral with regard to jurisdictional claims in published maps and institutional affiliations.

Copyright: (C) 2022 by the authors. Licensee MDPI, Basel, Switzerland. This article is an open access article distributed under the terms and conditions of the Creative Commons Attribution (CC BY) license (https:// creativecommons.org/licenses/by/ $4.0 /)$.

\begin{abstract}
There is growing interest in the development of edible delivery systems to enrich, protect and release bioactive compounds within foods. Emulsion-based systems are a good strategy for this purpose. Considering that chia oil (high levels of omega-3 fatty acids) is very susceptible to lipid oxidation, conventional and bilayer $\mathrm{O} / \mathrm{W}$ emulsions were studied as a function of refrigerated storage. Monolayer emulsions were stabilized with deoiled sunflower lecithin while, in the case of bilayer ones, chitosan was also added by applying the electrostatic deposition technique. Bilayer emulsions presented a monomodal droplet size distribution while a shoulder towards larger particle sizes appeared for the conventional systems. Some signs of destabilization by the creaming process were recorded for monolayer emulsions, instead of the high stability associated with the other ones. The presence of chitosan significantly affected the rheological characteristics of emulsions by increasing their viscosity and modifying their flow behavior. In terms of oxidative stability, bilayer emulsions recorded the lowest PV values during the refrigerated storage and represent a better protective system than other ones included in the bulk oil. Thus, bilayer emulsions are a suitable option for the delivery of chia omega-3 and other PUFAs, with potential application in the food industry.
\end{abstract}

Keywords: chia by-products; electrostatic layer-by-layer deposition; modified sunflower lecithin; mono and bilayer $\mathrm{O} / \mathrm{W}$ emulsions; omega-3 fatty acids

\section{Introduction}

Emulsions with enhanced stability against environmental stresses and release properties can be obtained by applying an interfacial engineering technique layer by layer (LBL) based on the electrostatic deposition of charged biopolymers onto droplet surfaces with opposite charge. These systems result in oil droplets being stabilized by multiple interfacial layers constituted by an emulsifier layer and one or more biopolymer ones. Different emulsion characteristics such as charge, thickness, and composition, as well as bulk physicochemical properties, can be affected by this interfacial technology. Thus, the LBL technique could be used for the food industry to develop functional food with improved stability during manufacture, storage, transport, and utilization [1]. Because of the high omega-3 content of chia oil and its susceptibility to lipid oxidation, its inclusion into multilayer systems using a natural emulsifier such as sunflower lecithin-a by-product of the oil-degumming process—and chitosan - a waste product of the marine food processing industry-is of interest.

The aim of this research work was to develop chia O/W mono- and bilayer emulsions, whilst evaluating their physicochemical characteristics as a function of refrigerated storage to obtain interesting information about their potential application as omega-3 delivery systems. 


\section{Materials and Methods}

\subsection{Materials}

Chia oil was provided by Nutraceutica Sturla SRL (Buenos Aires, Argentina). Fatty acid composition was determined by GC according to IUPAC, 1992 [2], resulting in 10.5, 2.5, 6.1, 19.5 and 61.3\% for C16:0, C18:0, C18:1, C18:2, C18:3, respectively. Chitosan (Ch) of medium MW $250 \mathrm{kDa}$ and deacetylation of 75-85\% was purchased from Sigma Chemical Company (St. Louis, MO, USA) and sunflower lecithin was provided by Vicentin SAIC (Santa Fe, Argentina). All reagents were analytical grade.

\subsection{Methods}

\subsubsection{Sunflower Lecithin Modification}

The deoiling process of native sunflower lecithin was carried out according to American Oil Chemists' Society Official Method Ja 4-46 [3].

\subsubsection{Preparation of Emulsions}

A primary emulsion (L) with 5\% (wt/wt) of chia seed oil and deoiled sunflower lecithin (DSL) was obtained in two homogenization steps using an Ultraturrax T-25 (Janke and Kunkel GmbH, Staufen, Germany) (9500 rpm, $1 \mathrm{~min}$ ) and a high-pressure valve homogenizer (Niro Soavi, Parma, Italy) (600 bar, 4 passes) at $\mathrm{pH}$ 3. From the monolayer system, a secondary emulsion ( $\mathrm{LCh}$ ) was obtained through the addition of $0.2 \%$ chitosan. In order to prevent microbial growth both nisine $0.0012 \%(\mathrm{wt} / \mathrm{wt}$ ) and potassium sorbate $0.1 \%(w t / w t)$ were added. Mono- and bilayer systems were stored 30 days at $4{ }^{\circ} \mathrm{C}$.

\subsubsection{Droplet Size}

The droplet size distribution curves (DSD) and the $\mathrm{D}_{[3,2]}$ mean diameter were determined using a particle size analyzer Malvern Mastersizer 2000E (Malvern Instruments Ltd., Worcestershire, UK).

\subsection{4. ¿-Potential}

The $\zeta$-potential measurements were performed with a Zeta Potential Analyzer Brookhaven 90 Plus/Bi-MAS (Brookhaven Instruments Corporation, Holtsville, NY, USA) in a range -100 to $50 \mathrm{mV}$ according to Julio et al. [4].

\subsubsection{Rheological Properties}

Rheological measurements were carried out at $25 \pm 1{ }^{\circ} \mathrm{C}$ using a rheometer Haake RS600 (Haake, Germany) with a coarse plate-plate sensor system according to Julio et al. [4].

\subsubsection{Emulsion Stability}

Emulsions' global stability was monitored by the evolution of their backscatter profiles using a Vertical Scan Analyzer Quick Scan (Coulter Corp., Miami, FL, USA) according to Pan et al. [5]. Samples were transferred to cylindrical glass tubes and measured periodically for 30 days.

\subsubsection{Peroxide Value (PV)}

The primary products of lipid oxidation were determined according to Shantha and Decker [6] as a function of refrigerated storage.

\section{Results and Discussion}

Figure 1 shows the schemes of the monolayer (L) and bilayer (LCh) systems with their respective $\zeta-$ potential values at $\mathrm{pH} 3$. The electrostatic deposition of cationic chitosan onto the DSL-stabilized oil droplets was evidenced thought the inversion charge. In this sense, the droplet surface charge of monolayer emulsions of $-37 \mathrm{mV}$ turned to $+46 \mathrm{mV}$ for bilayer emulsions because of chitosan deposition. These results could be due to the opposite 
charges between DSL and chitosan molecules at this $\mathrm{pH}$ level, both being compounds mutually attracted.

Monolayer emulsion: L

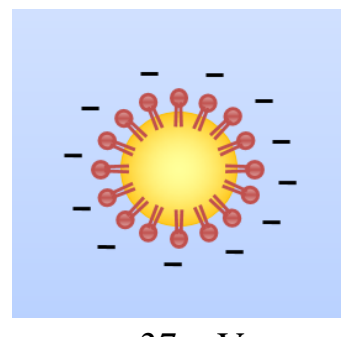

$-37 \mathrm{mV}$

\section{Bilayer emulsion: $\mathbf{L C h}$}

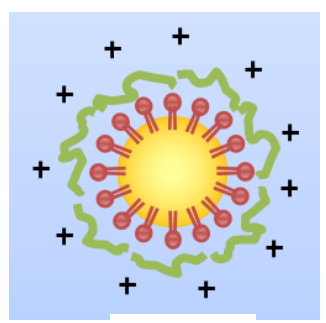

$46 \mathrm{mV}$

Figure 1. Schemes of chia oil droplets from mono- and bilayer emulsions and their $\zeta$-potential values.

Bilayer emulsions presented a monomodal DSD curve while a shoulder corresponding to a second population of larger droplet sizes was observed in DSD from monolayer systems (data not shown); $\mathrm{D}_{[2,3]}$ values for $\mathrm{L}$ and $\mathrm{LCh}$ emulsions were 0.32 and $0.26 \mu \mathrm{m}$, respectively. No significant $(p>0.05)$ changes in particle size were recorded during refrigerated storage.

The flow curves of the different systems were obtained from the rheological data fitting to the Power Law model equation $\left(R^{2}>0.99\right)$. The apparent viscosity values of emulsions at $100 \mathrm{~s}-1(\eta 100)$ - typical shear rate for food processes, such as stirring or mastication, flow through a pipe-were also calculated [7]. According to Figure 2, monolayer systems behaved as Newtonian fluids $(n \sim 1)$, while the chitosan deposition into bilayer emulsions led to shear-thinning behavior $(n<1)$. Additionally, a significant increase $(p<0.05)$ in $\eta_{100}$ was recorded, resulting in 1.54 and $9.97 \times 10^{-3}$ Pa.s ${ }^{n}$ for L and LQ systems, respectively.

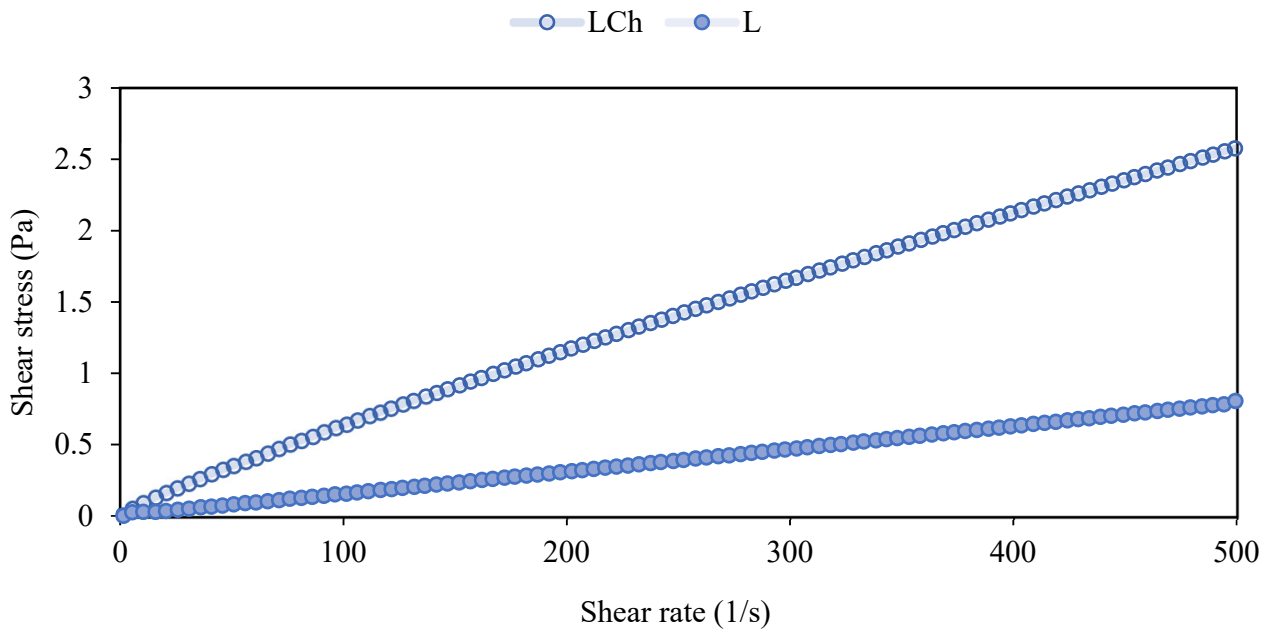

Figure 2. Flow curves of mono- and bilayer emulsions with chia oil.

Regarding global stability, primary emulsions experienced creaming destabilization, which can be seen from their BS profiles. In contrast, secondary systems were more stable, with their BS profiles remaining unchanged over the storage period. This fact could be associated with the higher aqueous phase viscosity of bilayer emulsions, which would reduce the oil droplets' movement (Figure 3). 

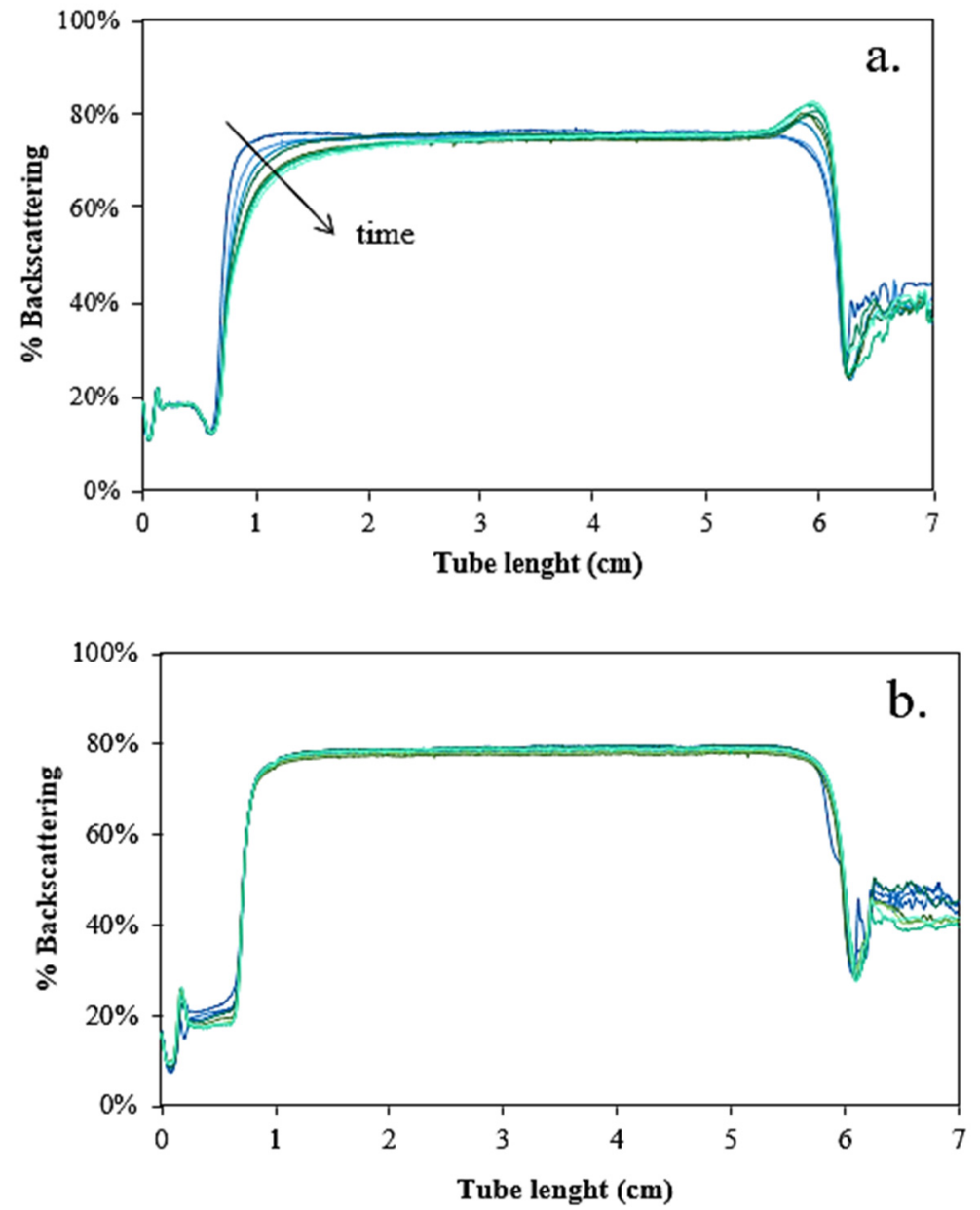

Figure 3. Back scattering profiles (\%BS vs. tube length) of mono- (a) and bilayer (b) chia $\mathrm{O} / \mathrm{W}$ emulsions during refrigerated storage.

Bilayer emulsions with DSL and chitosan presented a lower PV and, therefore, higher oxidative stability than bulk oil and monolayer systems after 30 days of refrigerated storage (Figure 4). This fact indicates the positive effect of the bilayer formed around the oil droplets to improve the protection against lipid oxidation. Some authors suggest that chitosan could act as a free radical scavenger, while others indicate that positively charged droplets could repel $\mathrm{Fe}^{+}$ions, reducing lipid oxidation [8].

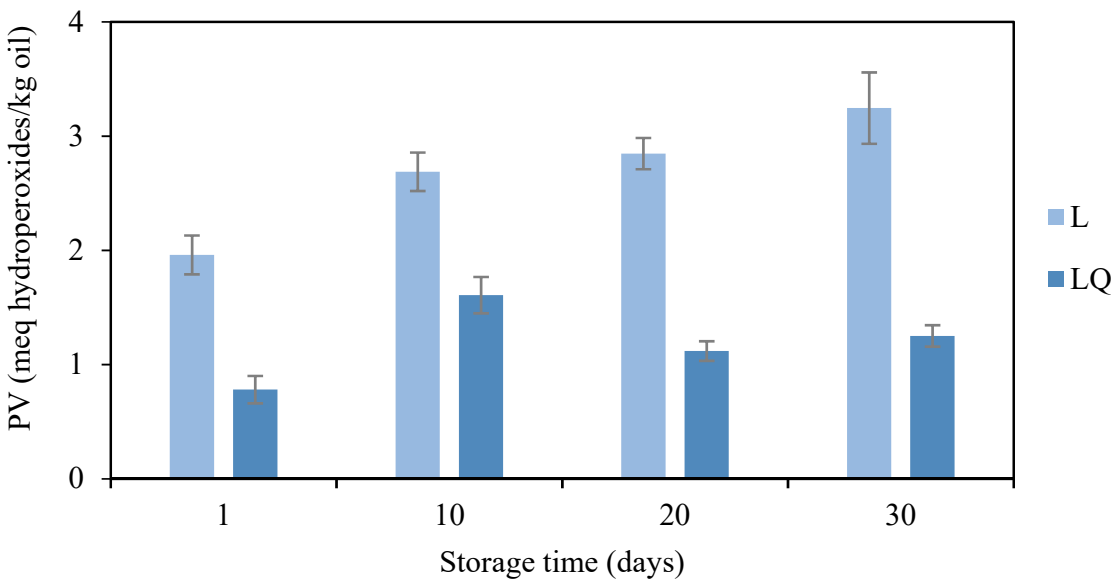

Figure 4. Evolution of hydroperoxides values of mono- and bilayer chia $\mathrm{O} / \mathrm{W}$ emulsions at 1, 10, 20 and 30 days of refrigerated storage. Average values are shown $(n=2)$. 


\section{Conclusions}

Mono- and bilayer emulsions containing chia oil were developed by applying the layerby-layer technique. The electrostatic deposition of chitosan onto a DSL interfacial membrane to form a bilayer around the chia oil droplets was evidenced through the inversion charge at $\mathrm{pH} 3$.

Bilayer emulsions presented smaller and monodisperse oil droplets and a better longterm stability than conventional systems. Additionally, bilayer emulsions provided higher protection against chia oil oxidation, recording lower PV values over the storage period. From these results, the bilayer emulsion proved to be an effective system to protect and deliver of omega-3 fatty acids from chia oil into functional foods.

Funding: This research received no external funding.

Institutional Review Board Statement: Not applicable.

Informed Consent Statement: Not applicable.

Data Availability Statement: Not applicable.

Acknowledgments: This work was supported by grant Ia ValSe-Food-CYTED (119RT0567) Spain, Universidad Nacional de La Plata (UNLP) (11/X907), and the ANPCyT (PICT 2016-0323), Argentina.

Conflicts of Interest: The authors declare no conflict of interest.

\section{References}

1. Klinkesorn, U.; Sophanodora, P.; Chinachoti, P.; McClements, D.J.; Decker, E.A. Stability of Spray-Dried Tuna Oil Emulsions Encapsulated with Two-Layered Interfacial Membranes. J. Agric. Food Chem. 2005, 53, 8365-8371. [CrossRef] [PubMed]

2. IUPAC. Standard methods for the analysis of oils, fats and derivates. In International Union of Pure and Applied Chemistry, Oxford, 7th ed.; Paquot, C., Hautfenne, A., Eds.; Blackwell Scientific Publications Inc.: Oxford, UK, 1992.

3. AOCS. Official Methods and Recommended Practices of the American Oil Chemists' Society, 4th ed.; AOCS Press: Champaign. IL, USA, 1994.

4. Julio, L.M.; Copado, C.N.; Diehl, B.W.; Ixtaina, V.Y.; Tomás, M.C. Chia bilayer emulsions with modified sunflower lecithins and chitosan as delivery systems of omega-3 fatty acids. LWT 2018, 89, 581-590. [CrossRef]

5. Pan, L.G.; Tomás, M.; Añon, M.C. Effect of sunflower lecithins on the stability of water-in-oil and oil-in-water emulsions. J. Surfactants Deterg. 2002, 5, 135-143. [CrossRef]

6. Shantha, N.C.; Decker, E. Rapid, Sensitive, Iron-Based Spectrophotometric Methods for Determination of Peroxide Values of Food Lipids. J. AOAC Int. 1994, 77, 421-424. [CrossRef] [PubMed]

7. McClements, D.J. Food Emulsions: Principles, Practices, and Techniques; CRC Press: Boca Raton, FL, USA, 2004.

8. Klinkesorn, U.; Sophanodora, P.; Chinachoti, P.; McClements, D.J.; Decker, E.A. Increasing the Oxidative Stability of Liquid and Dried Tuna Oil-in-Water Emulsions with Electrostatic Layer-by-Layer Deposition Technology. J. Agric. Food Chem. 2005, 53, 4561-4566. [CrossRef] [PubMed] 